\title{
Nivel de depresión del adulto mayor de la Casa de día de la Cruz de Mangas, Tezontepec de Aldama
}

\author{
Main Depression level of the elderly in the Day House of the Cross of Mangas, Tezontepec de \\ Aldama.
}

\section{Maria del Pilar Hernández-Reyes ${ }^{a}$, Luz V. Reyes-Porras ${ }^{b}$, Benjamín López-Nolasco ${ }^{c}$, Ariana Maya-Sánchez ${ }^{d}$, Gabriela Maldonado-Muñiz ${ }^{e}$}

\begin{abstract}
:
According to the World Health Organization, more than $20 \%$ of the population aged 60 and over suffer from a mental or neuronal disorder, with dementia and depression being the most frequent neuropsychiatric conditions. Objective: To evaluate the level of depression presented by the elderly. Material and method: This is a quantitative, descriptive study, $\mathrm{n}=17$ elderly people, enrolled in the Day House, who agreed to participate; The Yesavage Test geriatric depression scale (alpha coefficient of 0.74) was applied, in a period of April 2019. I have the approval of the ethics committee and signature of informed consent. Results: The average age was 69.41 years, $29.4 \%$ of the female gender, $70.6 \%$ male gender, $47.1 \%$ widowers, $47.1 \%$ dedicated to shepherding. The level of depression obtained $41.17 \%$ normal, $52.9 \%$ level and 5.8\% moderate. Conclusions: The level of depression is present at this stage of life when it is at a normal level.
\end{abstract}

\section{Keywords:}

Senior Adult, Aging, Depression

\begin{abstract}
Resumen:
De acuerdo con la Organización Mundial de la Salud (OMS), de la población adulta mayor mayor de 60 años el $20 \%$ sufre algún trastorno mental o neural, siendo la demencia y la depresión los padecimientos neuropsiquiátricos más frecuentes. Objetivo: Evaluar el nivel de depresión que presenta el adulto mayor. Material y método: El presente es un estudio cuantitativo, descriptiva, $\mathrm{n}=17$ personas mayores, inscritos en la Casa de Día, que aceptaron participar; se aplicó la escala de depresión geriátrica Test de Yesavage (coeficiente alfa de 0.74 ), en un periodo de abril 2019. Conto con la aprobación del comité de ética y firma de consentimiento informado. Resultados: La edad media fue de 69.41 años, $29.4 \%$ de género femenino, $70.6 \%$ género masculino, $47.1 \%$ viudos, $47.1 \%$ dedica a pastorear. El nivel de depresión encontrado fue del $41.17 \%$ en estado normal, $52.9 \%$ leve y $5.8 \%$ moderado. Conclusiones: El nivel de depresión está presente en todas las etapas de la vida a un cuándo se encuentra en un nivel norma, e interfieren vida de las personas.
\end{abstract}

\section{Palabras Clave:}

Adulto Mayor, Envejecimiento, Depresión

\section{Introducción}

\author{
a Autor de Correspondencia, Universidad Autónoma del Estado de Hidalgo Escuela Superior de Tlahuelilpan, Alumnos de la Licenciatura \\ en Enfermería, Email: hdez360528@uaeh.edu.mx \\ b Universidad Autónoma del Estado de Hidalgo Escuela Superior de Tlahuelilpan, Alumnos de la Licenciatura en Enfermería, Email: \\ vale362429@uaeh.edu.mx \\ c Universidad Autónoma del Estado de Hidalgo Escuela Superior de Tlahuelilpan, Profesor Investigador, Email: lopez8496@uaeh.edu.mx \\ d Universidad Autónoma del Estado de Hidalgo Escuela Superior de Tlahuelilpan, Profesor por asignatura de la Licenciatura en Enfermería \\ Email: arianamaya_8228@uaeh.edu.mx
}

e Universidad Autónoma del Estado de Hidalgo Escuela Superior de Tlahuelilpan, Profesor Investigador, Email: gmaldonado@uaeh.edu.mx 
El presente trabajo tuvo como objetivo de Analizar el nivel de depresión que presento el adulto mayor en la Casa de Día de la Cruz de Mangas, Tezontepec de Aldama, ya que en cuestión con los datos publicados por organismos internacionales y nacionales el nivel de depresión tiende a incrementar en esta etapa de la vida, la depresión es una enfermedad que probablemente sea la principal causa de sufrimiento en los adultos mayores con la que se ve afectada directamente su calidad de vida; se encontró que existen diversos estudios realizados en América Latina que muestran la tendencia del aumento de depresión en esta etapa de la vida.

La Organización Mundial de la Salud (OMS), menciona que, de los adultos mayores de 60 años, más del $20 \%$ sufre algún trastorno mental, teniendo en cuenta que la depresión y la demencia encabezan la lista de los padecimientos (CONAPO,2017). La depresión tiende a afectar al $7 \%$ de la población mundial de los adultos mayores, seguido por la demencia con un $5 \%$ de afectación (OPS,2013) en el estado de hidalgo se ha visto un incremento de la depresión por cerca del $29 \%$ en la población de adultos mayores, y esta es mayor en comparación con el porcentaje que reportan los estado de Querétaro, Morelos y Tlaxcala (AM Hidalgo, 2018), las características que destacan en este grupo de edad para incrementar el índice de depresión está altamente relacionadas con la edad, sexo, el estado civil, la ocupación o en que dedican su tiempo, las relaciones sociales, etc. (De Los Santos, 2018)

\section{Adulto Mayor}

La persona mayor es un ser socialmente frágil en el sentido de que suele haber perdido algunos de los pilares sobre los que se sostenía (familiares, amigos, actividad profesional e independencia económica), lo cual la pone en riesgo de caer en desequilibrios psíquicos y en trastornos depresivos (Can Valle, 2017)

\section{Envejecimiento}

El envejecimiento es la consecuencia de la acumulación de una gran variedad de daños moleculares y celulares a lo largo del tiempo, lo que conlleva un descenso de las capacidades físicas y mentales, un aumento del riesgo de enfermedad, y finalmente a la muerte. Dentro de las patologías que suelen incluirse en esta etapa cabe mencionar la pérdida de la audición, cataratas, dolores de espalda, cuello y osteoartritis, neuropatías obstructivas crónicas, diabetes, depresión y demencia (Munoz, 2017)

\section{Depresión}

La depresión a pesar de ser un trastorno mental, puede ser tratada con actividades como la terapia ocupacional entre otras en el primer nivel de atención por personal no especialista pero sí que forme parte del personal de salud. Cuando esta no es diagnosticada o tratada a tiempo, avanza como lo hacen las demás enfermedades a niveles considerables (cónica o recurrente) y puede interferir de manera impoertant5e con las actividades de la vida diario de la personal, por lo que podría hablarse de una afectación global psíquica y física, haciendo especial énfasis en la esfera afectiva (Guía de Práctica Clínica, 2015)

\section{Planteamiento del problema}

En América latina la Organización Panamericana de la Salud (OPS) en coordinación con la Organización Mundial de la Salud (OMS), determinan que cerca del $15 \%$ de la población de adultos mayores sufres o padecen de algún trastorno mental, teniendo en cuenta que la depresión y la demencia encabezan esta lista, en consideración con él situación biológica y social que experimentan las personas y los mecanismos de defensa que tiene el cuerpo humano. Conforme a las proyecciones que estimo el Consejo Nacional de Población (CONAPO, 2017), en el país residen cerca de 12973411 personas de 60 y más años, de los cuales $53.9 \%$ son mujeres y $46.1 \%$ son hombres (CONAPO, 2015). La depresión afecta a nivel mundial al $7 \%$ de ellos y la demencia al $5 \%$. En cuanto a la estructura por sexo, se hace más evidente un mayor monto de mujeres, consecuencia de una sobre mortalidad masculina que se agudiza en esta etapa de vida: 
en la población de 60 a 64 años, hay 108 mujeres por cada 100 hombres y aumenta a 130 en el grupo de 80 y más años (CONAPO, 2014).

\section{Conocimientos previos}

De los Santos, Perla Vanessa., y colaboradores, presenta su estudio Prevalencia de depresión en hombres y mujeres mayores en México y factores de riesgo, en el año 2018, de la Universidad de Costa Rica, MUESTRA QUE existe una prevalencia de depresión en personas mayores de $74.3 \%$, incluyendo factores como la edad, el estado civil, actividades sociales, las cuales se incluyen como principales determinantes para desarrollar esta enfermedad en las personas mayores (De Los Santos, 2018).

Vilchez-Cornejo, Jennifer., y colaboradores, en su estudio Asociación entre trastorno depresivo y deterioro cognitivo en ancianos de tres ciudades del Perú, Lima, Perú en 2017, en donde indica que los índices de prevalencia de depresión se ven en aumento conforme la edad de un apersona avanza y se incluyen los trastornos neurocognitivos asociados a la depresión severa (VILCHEZ, 2017)

\section{Metodología del diseño o de estudio}

Este estudio es cuantitativo, aplicada, descriptivo, transversal, universo conformada por 60 personas mayores de 60 años, inscritos al a la Casa de Día. Se solicitó autorización al personal mediante un oficio donde se estableció el horario y el espacio en el cual se trabajó. Se elaboró y proporciono un consentimiento informado para su firma, previa explicación.

Se llevó a cabo la aplicación del instrumento escala de depresión geriátrica test de yesavage, consta de 15 ítems, mide el nivel de depresión geriátrica, las opciones de respuesta sí y no. el puntaje de corte de acuerdo a las puntuaciones obtenidas va de 0 - 5: normal, 6 - 10: depresión moderada y > 10: depresión severa.
La enfermería como disciplina Práctica Basada en la Evidencia Científica tiene el propósito de prestar un servicio a la persona, familia y la comunidad en general para mejorar, prevenir y realizar y técnicas de reinserción para una calidad de vida mejor (disminución de la depresión), en la presente investigación se pretende contar con datos específicos de la Casa de Día de la Cruz de Mangas, a fin de proponer intervenciones.

\section{Resultados}

La muestra estudio conformada por 17 adultos mayores:

Datos sociodemográficos: La media de la edad fue de 69.41 años, con un rango de $60-77 ; 29.4 \%$ de género masculino, $70.6 \%$ género femenino, $47.1 \%$ viudos, $41.2 \%$ casados, $41.2 \%$ no padecen ninguna enfermedad, en cuanto pasatiempos $47.1 \%$ pastorear, contaban con primaria completa $58.8 \%$.

El nivel de depresión se identificó que el $41.17 \%$ tuvo un nivel de depresión normal, el $52.9 \%$ un nivel de depresión leve y solo el $5.8 \%$ estuvo con un nivel moderado de depresión. (Ver tabla 1).

\section{Discusión}

Lazcano-Ortiz y colaboradores (2019), en la aplicación del Pre-test ninguno estuvo en el rango normal, depresión leve $67 \%$ (6 participantes) y el resto en depresión establecida 33\% (3 participantes), hacienda una comparación con nuestros resultados se obtuvo que el $41.17 \%$ tuvo un nivel de depresión normal, el 52.9\% un nivel de depresión leve y solo el $5.8 \%$ estuvo con un nivel moderado (Lazcano, 2019)

\section{Aplicación}




\begin{tabular}{ccc}
\hline Nivel de depresion & $f$ & $\%$ \\
\hline Normal & 7 & 41.17 \\
\hline Leve & 9 & 52.9 \\
\hline Moderada & 1 & 5.8 \\
\hline Grave & & \\
\hline Total & 17 & 100
\end{tabular}

Tabla 1. Frecuencia y porcentaje de depresión. Fuente: Instrumento Escala de depresión Geriátrica de Yesavage, n:17

\section{Conclusión}

El nivel de depresión de los Adultos mayores que asisten a la Casa de Dia de Tezontepec de Aldama, Hidalgo, se encuentra en niveles normales.

Mas sin en cambio puede estar influenciado por factores externos, los cuales dependerán de cada adulto mayor, por ello es de suma importancia que el profesional de enfermería, así como familiares y la sociedad en la que se desarrolla el adulto mayor, les brinde atención, cuidado y sean reconocido como parte de la sociedad y así evitar que sean aislados y discriminados por la misma.

\section{Referencias}

[1] INEGI. México: CONAPO; 2017 (28 de septiembre 2017; 30 de mayo del 2018. Disponible en: http://www.inegi.org.mx/saladeprensa/aproposito/201 7/edad2017_Nal.pdf

[2]Paho.org[Internet].Washinton:OPS;2013[actualiza da 10 de Oct 2013; citado 19 de Agosto 2018]. Disponible en: https://www.paho.org/hq/index.php?option=com_cont ent $\&$ view $=$ article $\&$ id $=9073: 2013$-depressiondementia-top-mental-disorders-people-over$60 \&$ Itemid $=1926 \&$ lang=es.
[3]

Am.com[Internet].Hidalgo:AmHidalgo;2018[actualiz ado 1 de enero 2018 ; citado 19 de Agosto 2018]. Disponible en: https://www.am.com.mx/2017/12/31/hidalgo/local/hid algo-ocupa-el-lugar-15-en-casos-de-depresion414719.

[4] De los Santos P.V., Carmona Valdés S.E. Prevalencia de depresión en hombres y mujeres mayores en México y factores de riesgo Población y Salud en Mesoamérica. Redalyc. 2018;15(2):91-114. http://www.redalyc.org/pdf/446/Resumenes/Resumen _44654575005_1.pdf.

[5] Can Valle A.R., Sarabia Alcocer B., Pacheco Quijano L.V. Depresión en mujeres adultas mayores y su afrontamiento. Dialnet.2017; 6(12):12-53. file:///C:/Users/woods/Downloads/Dialnet-

DepresionEnMujeresAdultasMayoresYSuAfrontamie nto-6121026.pdf

[6] Muñoz Muñoz C., La terapia ocupacional y la promoción de la salud emancipadora para personas mayores, Revista Chilena de Terapia Ocupacional, 2017 (19 de agosto del 2018); 17 (2); 111-117.

[7]Grupo de trabajo de Guía de Práctica Clínica Diagnostico Y tratamiento del Trastorno depresivo en el adulto. Publicado por el Instituto Mexicano del seguro social. Coordinación Técnica de Excelencia clínica; 2015. http://www.guiasalud.es/GPC/GPC_534_Depresion _Adulto_Avaliat_compl.pdf (Ultimo acceso 19 de agosto 2018).

[8] inegi.org. México; CONAPO: 2015;(6 de junio del 2017; 28 de septiembre 2017;31 de mayo del 2018) consultar en: http://www.inegi.org.mx/saladeprensa/aproposito/2 017/poblacion2017_Nal.pdf.

[9] inegi.org. México; CONAPO: 2014;(25 de septiembre de 2014; 31 de mayo del 2018) consultar en:

http://www.inegi.org.mx/saladeprensa/aproposito/2 014/adultos0.pdf 
[10] Vilchez Cornejo J., Soriano Moreno A., Saldaña Cabanillas D., Acevedo Villar T.H., Bendezú Saravia P., Ocampo Portocarrero b. Asociación entre trastorno depresivo y deterioro cognitivo en ancianos de tres ciudades del Perú. Scielo.2017;34(4):266-77. http://www.scielo.org.pe/pdf/amp/v34n4/a03v34n4.pd $\mathrm{f}$

[11] Lazcano-Ortíz M, Del Ángel-Monzón M, Huizache-Santos N, Islas-Muñoz JA, Lozada-Flores A, Sánchez-Escalona RA, Vega-Hernández DD, JiménezSánchez RC, Sánchez-Padilla ML. Efecto de la terapia ocupacional en el adulto mayor para disminuir la depresión. ICSA [Internet]. 5 de junio de 2019 [citado 1 de septiembre de 2019];7(14):43-9. Disponible en: https://repository.uaeh.edu.mx/revistas/index.php/ICS A/article/view/4435 